\title{
A MESH RE-ZONING TECHNIQUE FOR FINITE ELEMENT SIMULATIONS OF METAL FORMING PROCESSES
}

\author{
JUNG-HO CHENG ${ }^{\dagger}$ AND NOBORU KIKUCHI \\ Department of Mechanical Engineering and Applied Mechanics, The University of Michigan, Ann Arbor, Michigan, U.S.A.
}

\section{SUMMARY}

Based on some fundamental properties of finite element approximations, a mesh re-zoning scheme is proposed for finite element simulations of metal forming problems. It is demonstrated that this technique is indispensable in analysing many difficult forming processes, especially when there exist corners or very irregular shapes on the boundaries. The algorithm is tested by a backward extrusion process and direct extrusion through a square die.

\section{INTRODUCTION}

With a tremendous amount of effort and study, the applications of finite element methods to the analysis of large deformation problems have enjoyed a great deal of success. ${ }^{1}$ However, for many practical problems, obtaining reasonably accurate solutions is still questionable, if not impossible. For example, considering a backward extrusion process, an initial finite element model with the deformed mesh at 20 per cent reduction of the initial height is shown in Figure 1. It is clear that some of the elements around the corner of the punch distort severely, which make the deformed configuration a very bad reference state in the updated Lagrangian formulation.

In a recent paper, ${ }^{2}$ the authors presented an application and extension of the grid adaptation methods to improve the quality of finite element approximations in several metal forming processes. A result of the backward extrusion process by a combination of the $r$-method and the $h$ method, using the effective strain as the error measure, is reproduced in Figure 2. We concluded that although the grid adaptation was applied extensively, the difficulties that arose from the gap between the punch wall and the fold-over elements still existed. Excessive errors may also occur whenever sharp corners or very irregular boundary shapes are present in the large deformation analysis, in which the deformed configuration is, in general, considerably different from the stressfree one. A possible resolution to this problem, as has been demonstrated in References $3-6$, would be to use a mesh re-zoning technique.

The idea is to rediscretize a certain deformed configuration (e.g. at 5 per cent reduction of a backward extrusion process), which may be regarded as a new material, and all the historydependent quantities are properly transferred from the original model to the remeshed one. The computation is resumed from this new reference state. It is also necessary to design a scheme so that the deformed configurations can be displayed using the initial discretization. Depending on the problem, this re-zoning process may be repeated several times until the deformation is completed.

\footnotetext{
Now in the General Electric Company, Corporate Research and Development Center.
}

0029-5981/86/030219-10\$01.00

(C) 1986 by John Wiley \& Sons, Ltd.

Received 16 April 1985 


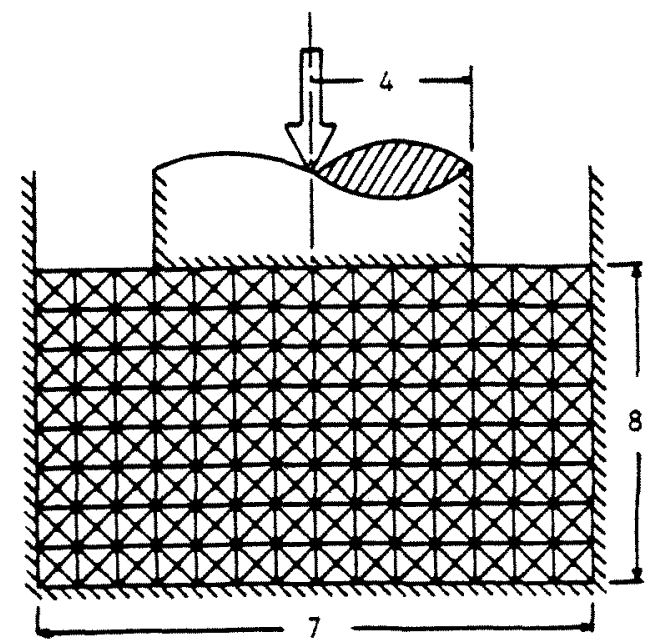

Material Constants:

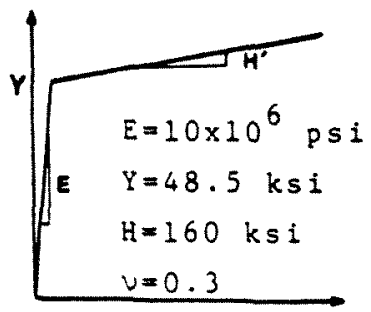

$20 \%$ REDUCTION

MAXIMUM VALUE OF $(1)=0.907 E+00$

MAXIMUM VALUE OF $(2)=0 \cdot 759 E+00$

MAX. EFFECT. STRAIN $=0.665 E+00$
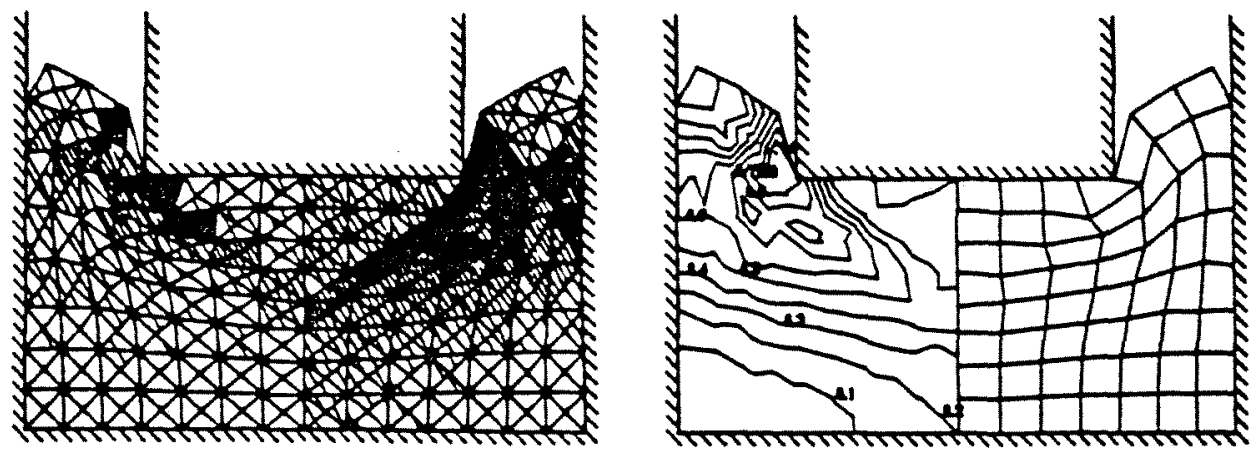

Figure 1. A backward extrusion process with an initial mesh and the deformed configurations at 20 per cent reduction; the shaded lines on the left-hand side represent the distributions of (1) effective strain at the right and (2) its difference among adjacent elements (left)

\section{$20 \%$ REDUCTION \\ MAX, EFFECT. STRAIN $=0 \cdot 185 E+01$}

Initiol Model
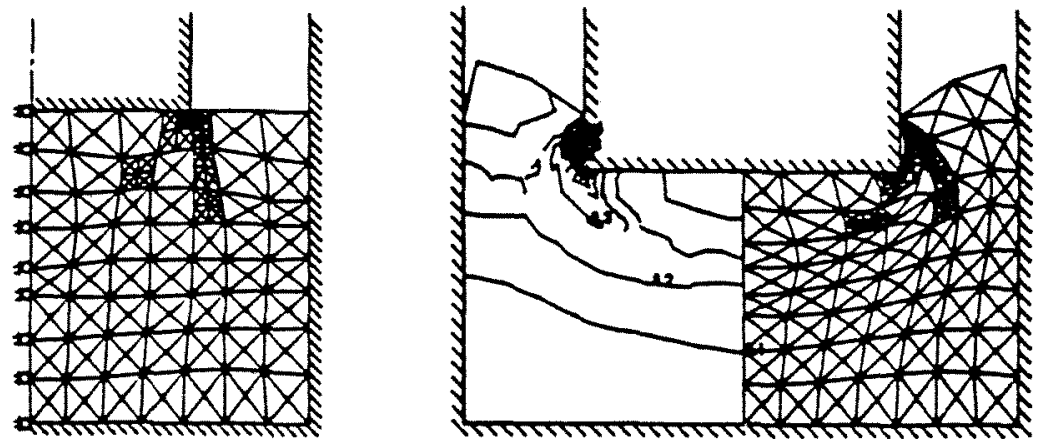

Figure 2. A combination of the $r$-method with the $h$-method to a backward extrusion process using the effective strain as the error indicator ${ }^{2}$ 
In the following, a simple yet effective method is proposed, based upon the fundamental characteristics of finite element approximation.

\section{REMESHING AND DISPLAYING ALGORITHMS}

Figure 3 shows a schematic diagram of transformation and interpolation of data from one mesh to another. In order to facilitate our discussions, a superscript 1 or 2 is attached to all the variables corresponding to the pertinent configuration. The first step is to distribute certain updated quantities to the nodal points. For constant-strain triangular elements, since the components of stresses and strains are obtained as constant values within each element, it is necessary to convert the elementwise quantities into the nodal ones. To do this, all the elements adjacent to any node $i$

\section{To distribute elementwise data to nodes}

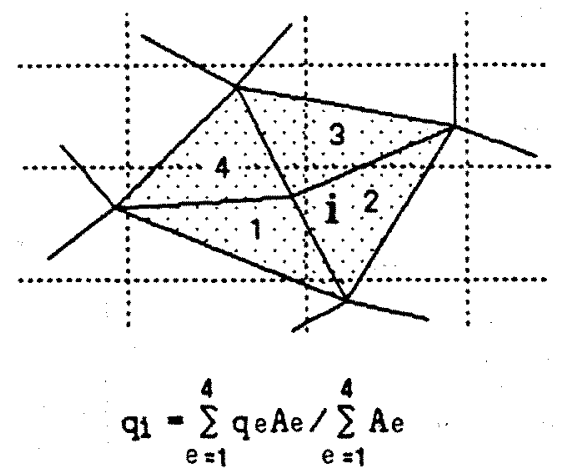

2. To transfer data from mesh 1 to mesh 2

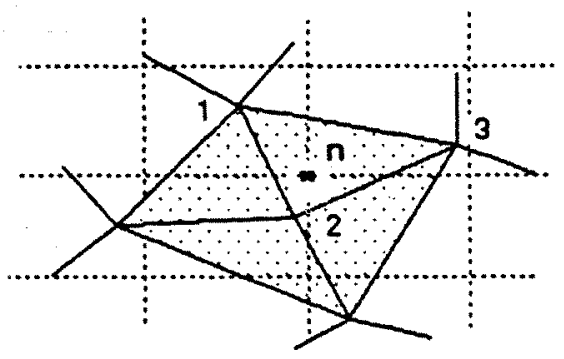

$$
q^{2} n=\sum_{\theta=1}^{3} q_{\theta}^{1} N 1_{8}\left(x_{n}\right)
$$

Figure 3. A schematic diagram of transformation and interpolation of data, $q$, from mesh 1 (solid lines) to mesh 2 (dotted lines), $A_{e}$ is the area of eth element and $N_{\beta}^{1}(\mathbf{x})$ are shape functions of mesh 1 
are identified and the value of $q_{i}$ is obtained by

$$
q_{i}=\sum_{e} q_{e} A_{e} / \sum_{e} A_{e}
$$

where $q_{e}$ is the value in the element $e$ whose area is denoted by $A_{e}$, and the summation is taken up to the total number of the elements around node $i$.

For higher order elements, since stresses and strains are evaluated at the Gauss points, the above process can be modified by solving a least square problem

with

$$
\operatorname{Min} 1 / 2 \int_{\Omega}\left(q-q^{*}\right)^{2} \mathrm{~d} \Omega
$$

and

$$
q=q_{\beta} N_{\beta}(\mathbf{x})
$$

$$
q^{*}=q_{\beta}^{*} M_{\beta}(\mathbf{x})
$$

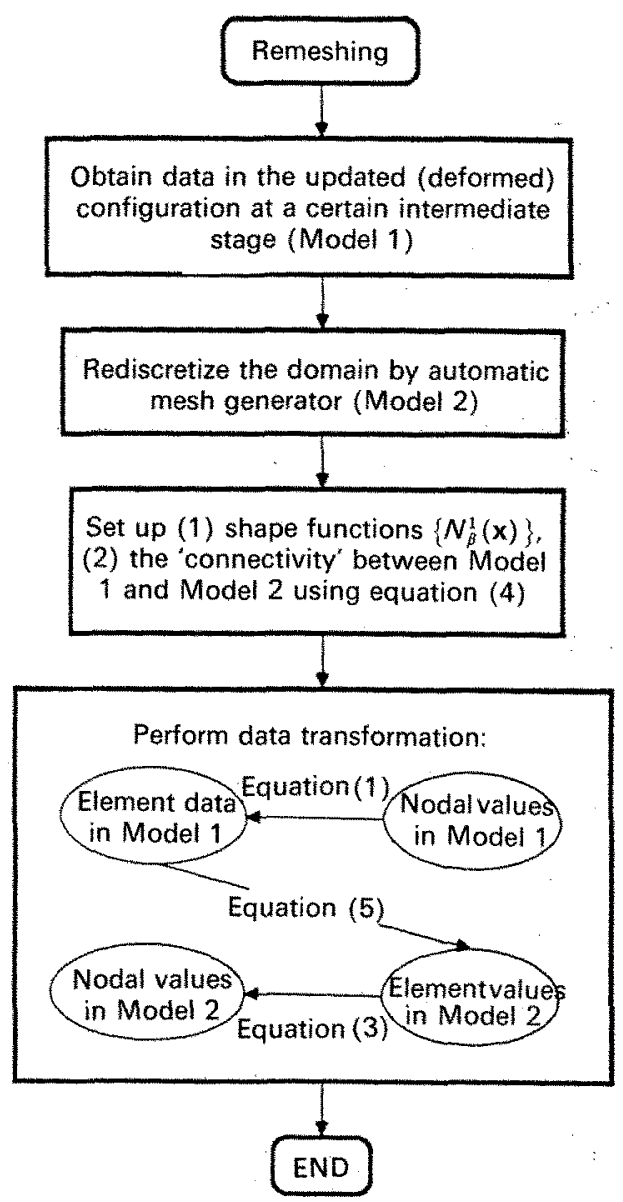

(a)

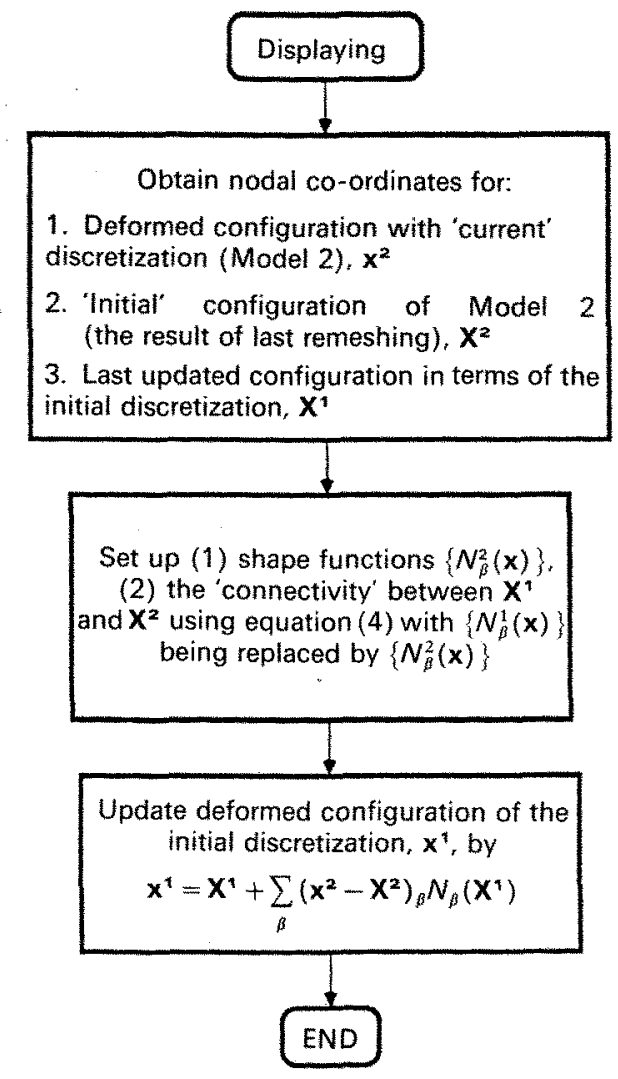

(b)

Figure 4. The re-zoning process: (a) remeshing; (b) displaying 
where $q_{\beta}$ are the nodal quantities to be determined, $N_{\beta}(\mathbf{x})$ are the conventional shape functions, and $q_{\beta}^{*}$ and $M_{\beta}(\mathbf{x})$ are, respectively, the known quantities and shape functions defined at the Gauss points.

Any quantity $q_{n}^{2}$ at the $n$th node of the second configuration can be obtained by the interpolation

$$
q_{n}^{2}=q_{\beta}^{1} N_{\beta}^{1}\left(\mathbf{x}_{n}\right)
$$

where $\left\{N_{\beta}^{1}(\mathbf{x})\right\}_{\beta=1, \mathrm{Ne}}$ is the set of area co-ordinates or shape functions defined in the first configuration, and $q_{\beta}^{1}$ is the value obtained at the $\beta$ th node (local numbering from 1 to the total number of the nodes, $N_{e}$ ) is an appropriate element.

To determine in which element of the 1 st configuration $\mathbf{x}_{n}$ is located (e.g. node $*$ in the shaded element of Figure 3), using the properties of shape functions we search for a node ' $n$ ' in the space ' 1 ' such that

$$
0 \leqslant N_{\beta}^{1}\left(\mathbf{x}_{n}\right) \leqslant 1, \quad \forall \beta=1, N_{e} .
$$

Basically, the procedures of remeshing and displaying are almost the same. The main difference lies in that it is the history-dependent values such as stresses and strains that are to be transferred to the rediscretized model when remeshing is considered, whereas only the updating of co-ordinates (by the displacements) is required in displaying the deformed configurations.

After the transformation (4) is made, the remeshing process is completed by obtaining the element wise value of $q_{e}^{2}$ from

$$
q_{e}^{2}=\left(\sum_{\beta=1}^{3} q_{\beta}^{2}\right) / 3,
$$

where $\beta$ takes the local numbering of the eth element in the second discretization.

The entire process of re-zoning can be summarized as shown in Figure 4.

\section{NUMERICAL EXAMPLES}

In this section, we shall present two example problems using the algorithms given in the previous section. Finite element formulations of large elastic-plastic deformation problems including unilateral contact conditions with friction are well established and applicable to many metal forming processes. ${ }^{7-12}$ In the following discussions, the solutions are obtained from the computer code 'EPAFRIC'1 developed by the authors to analyse various large deformation problems.

We start from a backward extrusion process shown schematically in Figure 5. The billet is assumed to be lead whose material properties are Young's modulus $E=36.54 \mathrm{GPa}$, Poisson's ratio $v=0.245$, yield strength $S_{Y}=23.44 \mathrm{MPa}$, and a very low strain hardening $H^{\prime}\left(=\mathrm{d} \bar{\sigma} / \mathrm{d} \bar{\varepsilon}^{p}\right)=$ $0.007 \mathrm{MPa}$. A constant coefficient of friction, $\mu_{F}=0.1$ is imposed on all the contact surfaces.

The billet is of initial diameter $50.8 \mathrm{~cm}(20 \mathrm{in}$.) and height $38.1 \mathrm{~cm}(15 \mathrm{in}$.). An axisymmetric case is assumed. Half of the domain is modelled by 1504 CST finite elements. The entire process up to 30 per cent reduction of the initial height is interrupted at every 5 per cent reduction for remeshing. Figure 6 demonstrate results of re-zoning at a typical step: remeshing at 10 per cent reduction and displaying the deformed mesh at 15 per cent reduction using the initial discretization in Figure 5. Several deformed configurations are shown in Figure 5, in which the diagonals of 4CST elements are omitted for the purpose of clear illustration. The applied loads given in Figure 7 are computed from the axial stresses in the elements adjacent to the punch.

The second problem is a plane-strain extrusion process through a square die, as shown in Figure 8. The material used is aluminium $1100-0$ with the following properties: $E=68.95 \mathrm{GPa}, v=0.32$, 

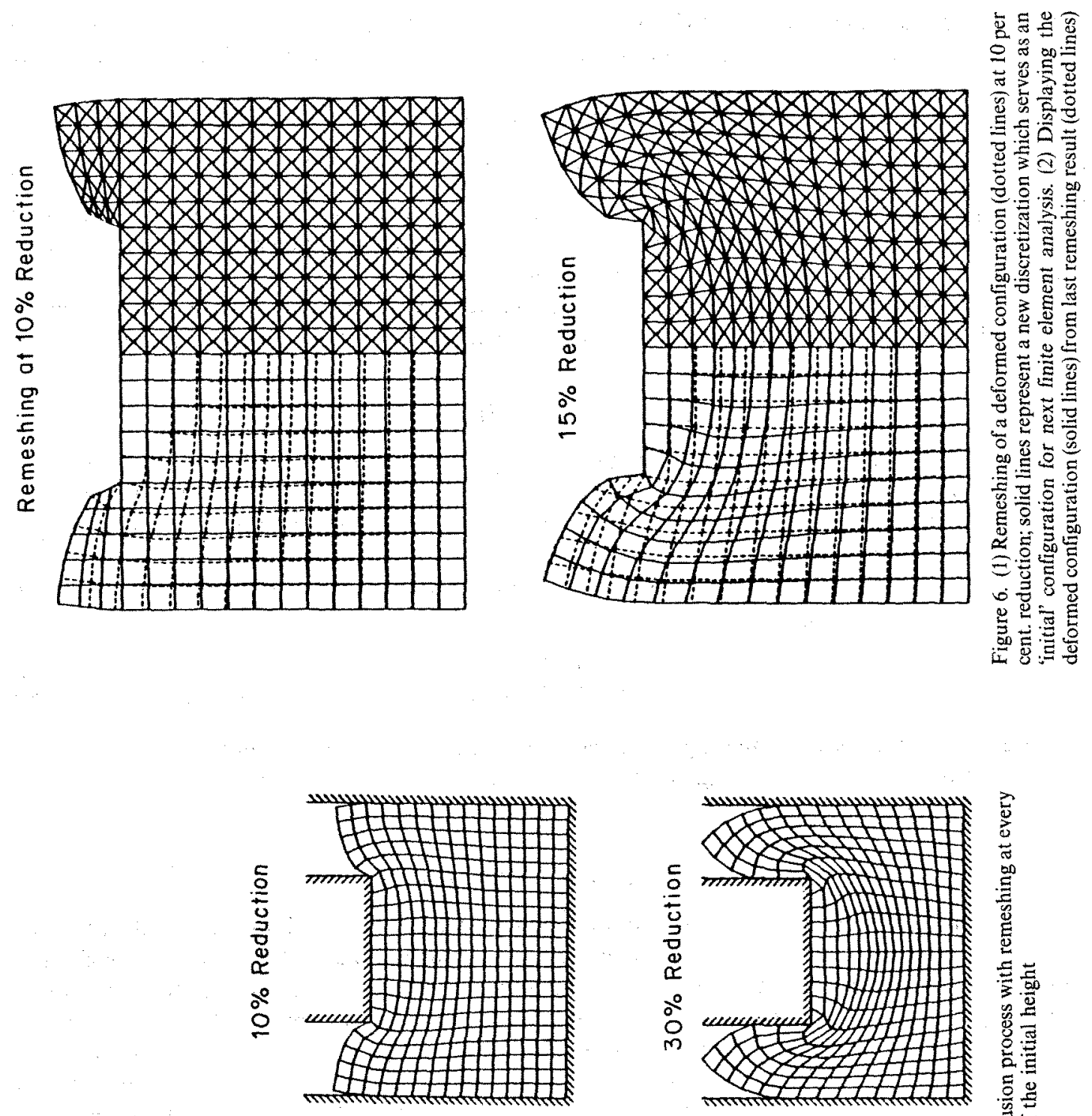

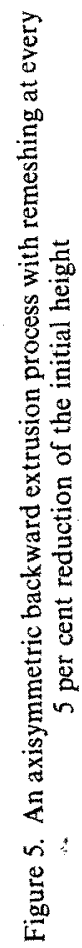
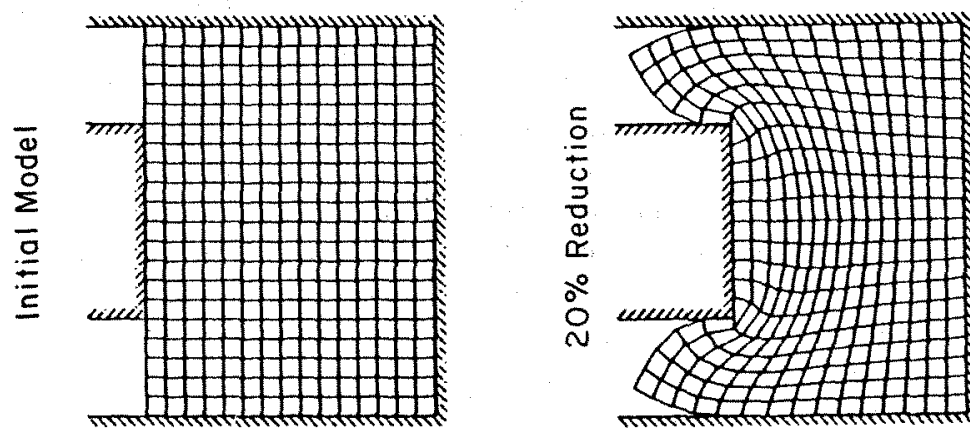


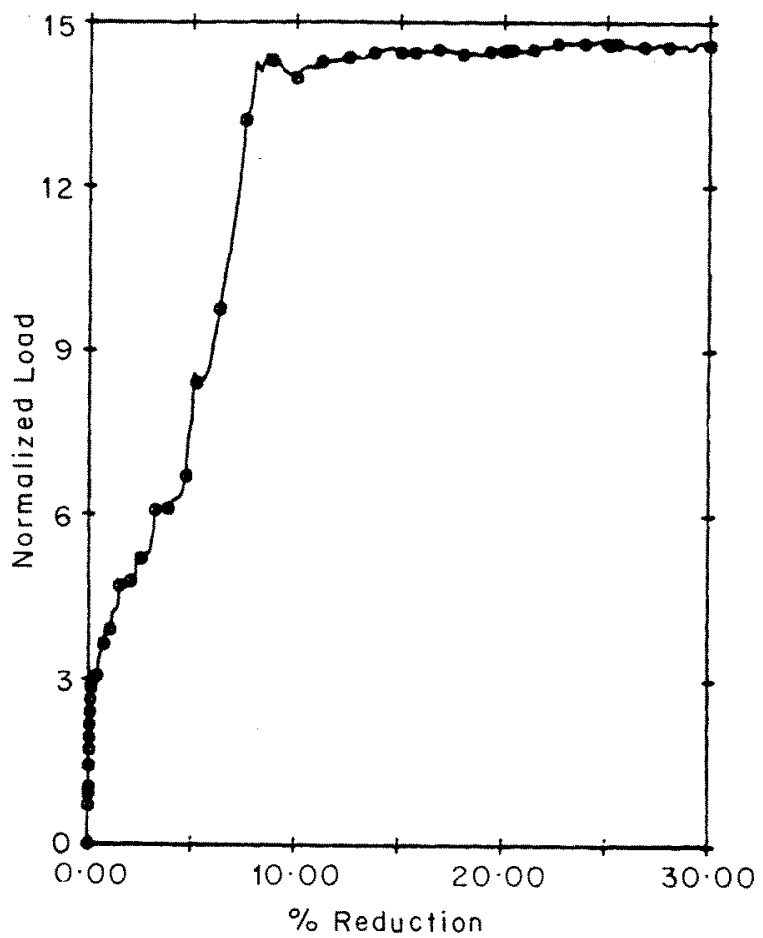

Figure 7. Applied load (normalized by $\pi R_{0}^{2} S_{Y}$ ) of a backward extrusion process by the re-zoning technique, where $R_{0}$ is the initial radius and $S_{Y}$ is the initial yield strength of the billet

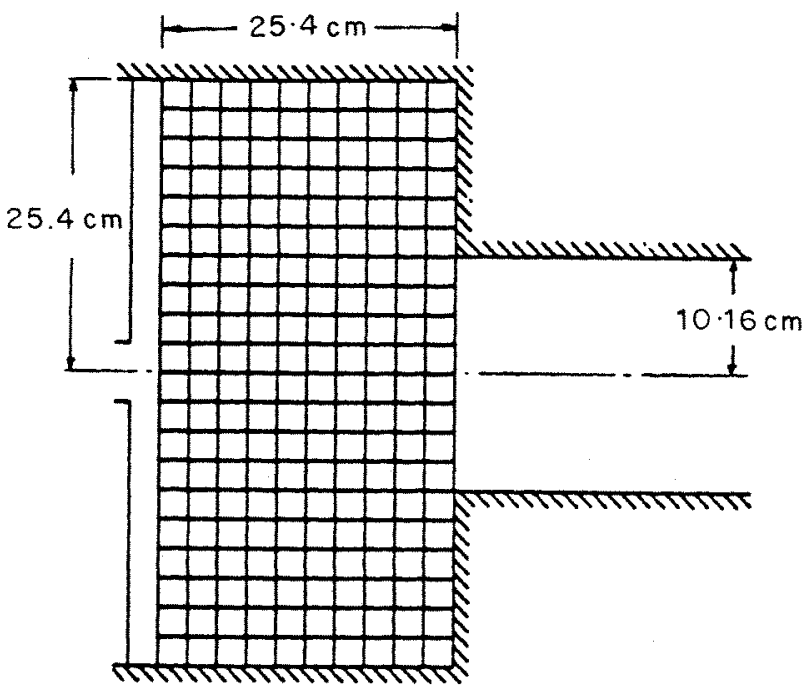

Figure 8. A schematic set-up for a plane-strain extrusion process through a square die 

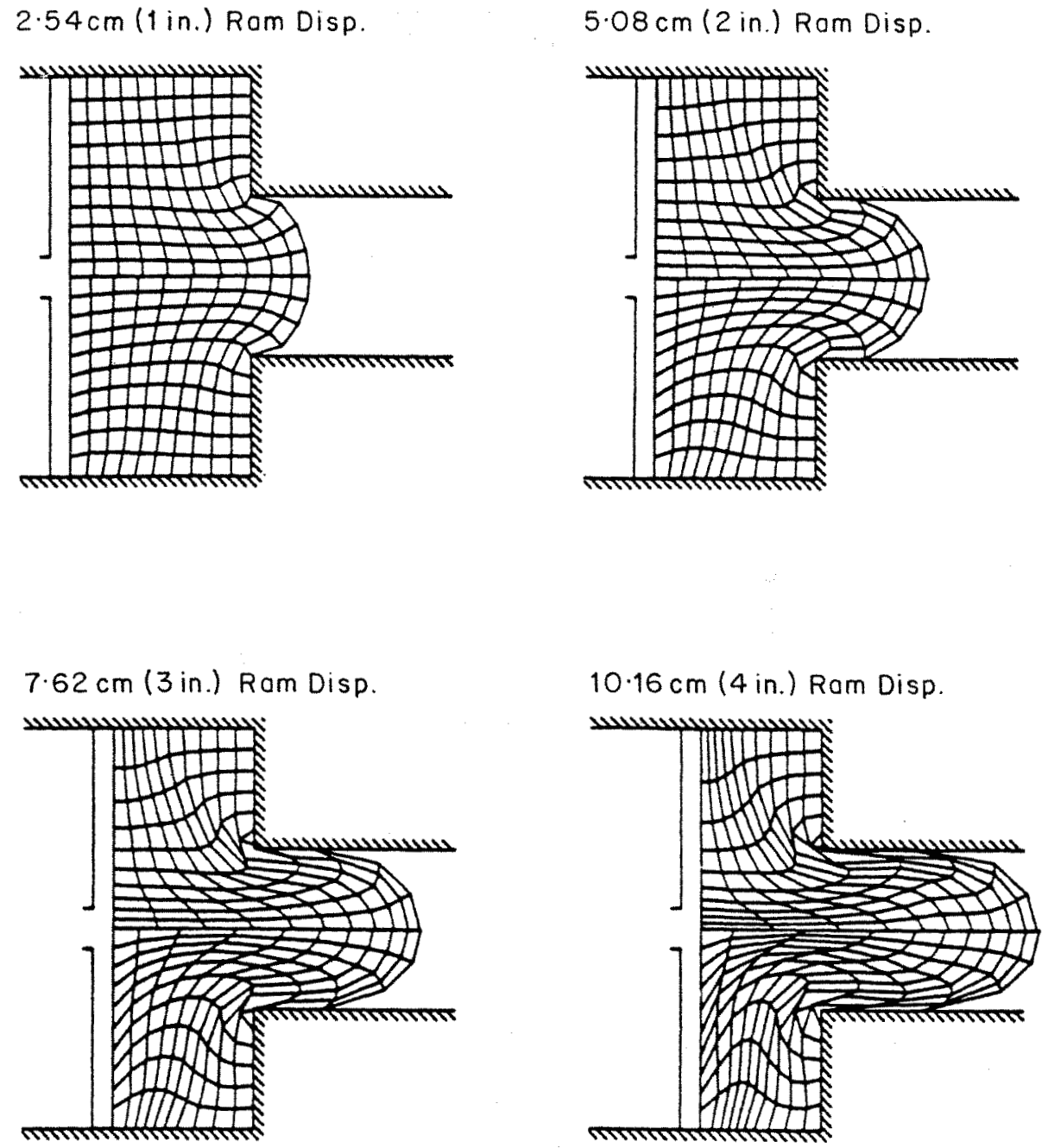

Figure 9. Deformed configurations of a plane-strain extrusion process through a square die; upper half for frictionless case, lower half for a constant $\mu_{F}=0.2$ assumed on all contact surfaces

yield strength $S_{Y}=31 \mathrm{MPa}$, and the post-yield behaviour $\bar{\sigma}=151 \cdot 7 \bar{\varepsilon}^{0 \cdot 25} \mathrm{MPa}$. The process is carried out by assuming either frictionless contact conditions or a frictional case with a constant coefficient of friction, $\mu_{F}=0 \cdot 2$, on all the contact interfaces. Deformed configurations for both cases are included together in Figure 9 for comparison purposes. Remeshing is performed at every $1.27 \mathrm{~cm}(0.5 \mathrm{in}$.) of ram displacement. In Figure 10 , the computed applied loads at increasing ram displacements are given to show the effect of 'smoothing' in evaluating the stresses. In general, when the process is resumed right after the deformed configuration is remeshed, the response of finite elements is very soft, as can be observed from the flat portions in the curves. However, the predicted load starts to ascent rapidly about $0.635 \mathrm{~cm}(0.25 \mathrm{in}$.) of ram displacement from the last intermittent step. It is expected that a very soft result will be obtained if the remeshing is made at each $0.635 \mathrm{~cm}$ of ram travel. The statement has to be justified by experimental work. More research efforts will be given toward this goal. 


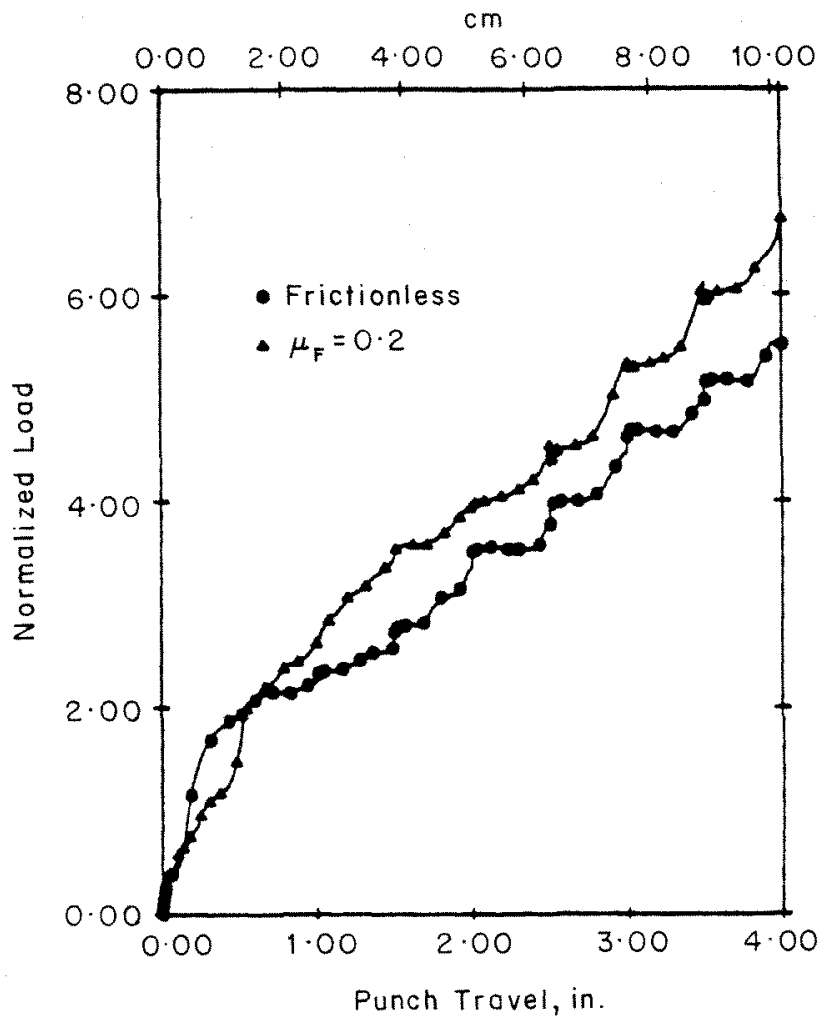

Figure 10. Applied loads (normalized by $\pi R_{0}^{2} S_{Y}$ ) vs. punch travel of a plane-strain extrusion process through a square die for two contact conditions

\section{ACKNOWLEDGEMENTS}

The authors are grateful to the National Science Foundation, Grant MSM-8410876 and the National Aeronautics and Space Administration, Grant NAG3-388, for the support associated with this project.

\section{REFERENCES}

1. J. H. Cheng and N. Kikuchi, 'An analysis of metal forming process using large deformation elastic-plastic formulations', to appear in Comp. Meths. Appl. Mech. Eng. (1984).

2. N. Kikuchi and J. H. Cheng, 'Adaptive remodeling of finite element grids for large deformation elastic-plasticity in metal forming analysis', to appear in Comp. Meths. Appl. Mech. Eng. (1984).

3. P. Hartley, C. E. N. Sturgess and G. W. Rowe, 'Computer simulation of metalforming process (elastic-plastic finiteelement analysis of forging)', Adv. Eng. Software, 4 (1) (1982).

4. K. Roll and Th. Neitzert, 'On the application of different numerical methods to cold forming processes', in Numerical Methods in Industrial Forming Processes (J. F. T. Pittman et al., Eds.), Pineridge Press, Swansea, U.K., 1982, pp. 97-107.

5. C. J. M. Gelten and A. W. A. Konter, Application of mesh-rezoning in the updated Lagrangian method to metal forming analyses', in Numerical Methods in Industrial Forming Processes, (J. F. T. Pittman et al., Eds.), Pineridge Press, Swansea, U.K., 1982, pp. 221-220.

6. A. A. K. Ai-Sened, P. Hartley, C. E. N. Sturgess and G. W. Rowe 'Forming sequences in axi-symmetric cold forging', pre-press (1984).

7. H. D. Hibbitt, P. V. Marcal and J. R. Rice, 'A finite element formulation for problems of large strain and large displacement', Int. J. Solids Struct., 6, 1069-1086 (1970). 
8. R. M. McMeeking and J. R. Rice, 'Finite-element formulation for problems of large elastic-plastic deformation', Int. J. Solids Struct., 11, 601-616 (1975).

9. K.-J. Bathe, E. Ramm and E. L. Wilson, 'Finite element formulations for large deformation dynamic analysis', Int. j. numer. methods eng., 9, 353-386 (1975).

10. J. H. Argyris and M. Kleiber, 'Incremental formulation in nonlinear mechanics and large strain elasto-plasticitynatural approach. Part I', Comp. Meths. Appl. Mech. Eng., 11, 215-247 (1977).

11. J. H. Argyris, J. St. Doltsinis, W. C. Knudson, J. Szimmat, K. J. William and H. Wustenberg, 'Eulerian and Lagrangian techniques for elastic and inelastic large deformation processes', in Computational Methods in Nonlinear Mechanics (J. T. Oden, Ed.) North-Holland, Amsterdam, 1980, pp. 13-66.

12. J. H. Cheng, 'Finite element simulations of metal forming processes by large deformation thermo-elasto-plastic formulation with grid adaptive and mesh rezoning method, Ph.D. dissert., Univ. of Michigan, Ann Arbor (1985). 